\title{
Neuroscience for Architecture: How Building Design Can Influence Behaviors and Performance
}

\author{
Andréa de Paiva \\ Fundação Getulio Vargas, FGV, Institute for Educational Development, São Paulo 01310-100, Brazil
}

\begin{abstract}
The purpose of this paper is to discuss recent findings in neuroscience that can be useful to architecture. Knowing the working patterns of the brain and how space affects cerebral functions can help architects design buildings that improve the user's behavior, performance and well-being. The built environment has a direct impact on the human brain. Social relations, focus, cognition, creativity, memory and well-being can be influenced by the surrounding physical space. Although it is not possible to create the perfect room, the space can be used in a strategic way, depending on the task that individuals are supposed to do there and depending on the people (age, gender, culture) who will make use of the space. Schools can be designed in a way to improve cognition, learning and memorization; hospital buildings can help improving recovery; workspaces can improve performance, creativity and collaboration. Above all, all spaces of long occupation should be designed in a way to improve well-being. How can architecture change automatic behaviors and nudge people to behave in a healthier way? Can architects create buildings and cities that improve socialization and happiness? Can criminality levels drop due to changes on the way the environments are designed? These are some of the questions that will be discussed in this paper.
\end{abstract}

Key words: Architecture, brain, neuroarchitecture, neuroscience, behavior, performance, well-being, design.

\section{Introduction}

"Architecture is the art which so disposes and adorns the edifices raised by man (...) that the sight of them contributes to his mental health, power and pleasure" [1].

Neuroscience is a field strongly associated with medicine. However, over the last few decades new technologies have helped boost neuroscientific research. Due to the several new findings in this area, neuroscience is now being applied in different fields, such as economy, marketing and leadership.

In economy, the idea of rationality in the process of decision making has always been defended. However, it has been proved recently that rationality does not play such an important role as it was believed. Decision making is much more influenced by impulsive, instinctive and affective thoughts than by logic and rationality.

Corresponding author: Andréa de Paiva, master of arts, research fields: neuroarchitecture: neuroscience applied to architecture and urban design.
In marketing this is also very explored nowadays. Professionals in this field try to create campaigns and advertisement announcements that incite impulsive, instinctive and affective reactions on their consumers. The way products are exposed and advertised is strategically thought aiming to foster impulsive purchases, boosting sales and profits.

Consumer loyalty can also be stimulated by the application of neuroscience to create the best marketing approach. Depending on the emotion generated by some kinds of stimuli, a strong bond can arise between client and brand.

In leadership, a leader that understands his team can adjust his way of leading in order to improve trust and flocking behavior, enhancing engagement and compromise among the team.

As a result, the number of professionals from different fields that are teaming up with brain scientists is increasing. This partnership allows new findings to be made and increases the practical application of neuroscience in several fields. 
For architecture, neuroscience can also be very helpful. Architects have always known the power of their buildings and how they can impact their users. The equation created by the psychologist Kurt Lewin (1890-1947) illustrates the role of the environment on individuals behavior: $\mathrm{B}=f(\mathrm{P}, \mathrm{E})$, which means behavior is a function between the Person (a unique individual with his own memories and genetics) and the Environment [2]. By Environment he means not only the social environment, but the physical environment too. Thus, behavior is also influenced by architecture. And this relation between environment and individual happens not only in a cognitive way, but also in an emotional or even instinctive way. "It turns out people have multiple subconscious tendencies and behaviors that govern their responses to built environments" [3].

According to neuroscience, the ability to process information consciously is less than $1 \%$ of the ability of unconscious processing. This means that most of the stimuli will affect individuals in a subconscious level. Therefore, even though people might be affected by it, they will not be aware of that.

The purpose of this paper is to discuss a few of the several matters that can be studied by this new science that arises from the partnership between neuroscientists and architects: neuroarchitecture (neuroscience applied to architecture). This paper also aims to point some of the benefits that neuroarchitecture can bring to the users of buildings and cities. The combination of neuroscience and architecture can be a great tool to help decipher some aspects of the relation between brain and space.

\section{Unconscious Processing of Information}

"In each of us there is another whom we don't know" [4].

The healthy brain is composed by systems in conflict. Sigmund Freud (1856-1939), the founder of psychoanalysis, came to that realization by talking with his patients and noticing their internal conflicts. As a consequence, he developed the psychic structure, comprising the id, ego and super-ego. The id (instinct) and the super-ego (moral) are completely opposites. Between them there is the ego, which tries to keep a balance between these two contrary instances.

Freud's findings, although having no direct somatic relation with the brain, inspired neuroscientists to study and realize that different patterns of behavior are controlled by specific structures in the brain.

Therefore, neuroscience seeks to map the human brain, understanding the multiple responsibilities of each structure. The simplest way to analyze the brain nowadays is to divide it in two systems: System I-fast thinking and System II-slow thinking [5].

System I is under the conscious level. It is fast, automatic, heuristic, implicit, intuitive, holistic, impulsive and emotive. It is the system responsible for controlling and monitoring the functions that keep the body safe (internally and externally). System II is conscious, slow, cognitive, systematic, explicit, analytic and reflexive. All human actions and reactions are guided by these two systems. However, they have different powers to influence behavior, decision making and performance.

System I is much faster than System II. In a tennis match, for example, when the adversary strikes the ball, the player does not have enough time to think consciously, to analyze the situation and plan how to reach the ball. All these happen in fractions of a second. The player's body basically reacts. It is an automatic behavior, controlled mainly by System I.

Another advantage of System I is that it needs less energy to work than System II. The human brain is hardwired to save energy. Conscious thoughts are tiring and need a lot of glucose. This is the reason why most brain processes are automatic. This automatization allows the brain to work faster and to save energy. If humans had to consciously think about most brain processes in order to realize them, the energetic cost —and the time cost—would be too high.

Lastly, System I can process much more information 
than System II. It controls at the same time the vital functions of the body (autonomic nervous system), automatic behaviors, and perceives the external environment. If individuals could consciously notice all stimuli from the spaces surrounding them, the brain would be overloaded. System I notices much more and selects only the relevant information to be processed by System II.

On the other hand, System II has a very logical intelligence that System I lacks. Most new situations are controlled by System II until it becomes automatic. Logical conclusions that need multiple factors analysis and long-term planning are also controlled by System II. System I, which is unconscious, automatic and impulsive, has a disposition for biased behaviors.

The relevance of System I in day-to-day life justifies seeing the brain as an iceberg: the small area visible above the water is the area of rationality and consciousness. But the big and long sharp bottom represents the proportion of automatic and unconscious behaviors that people are not aware of. David Rock [3] exemplifies this with the "elephant and the rider" metaphor: the conscious will is the rider that tries to control the larger and uncontrollable unconscious mind, the elephant. "With the prefrontal cortex taking up just $4 \%$ of total brain volume, modern brain science seems to affirm the truth of this metaphor. The prefrontal cortex, central to conscious decision making, has a degree of influence, but the rest of the brain is bigger and stronger" [3].

In conclusion, one of the greatest contributions from neuroscience to other fields of knowledge-including architecture - is the understanding that humans are hardwired to present much more impulsive, instinctive and emotive behaviors and perceptions than rational and conscious ones. With that in mind, an important question presents itself: Have architects been designing their buildings for System I or System II?

\section{Space, Message and Expectation}

"We shape our buildings; thereafter they shape us"
[6].

Architects have already been thinking about the messages their buildings send to their users. Medieval churches, with their long plans, high ceilings, and stained-glass windows at the top of the walls showed, through architecture, how great divine power can be. The impact of such message to individuals invoked respect and emotion. The classic CEO's room, located on the top floor of the company's building and decorated with marble and wood also shows power and invokes different behaviors on both, the owner of the room and the people who will visit him there. Thus, this communication between building and people is not new. The great difference is that it used to be done in a more intuitive and empiric way.

Neuroscience applied to architecture has proved that spaces can impact directly the way System I works. Since System I is unconscious, automatic and impulsive, a great part of such impact will not be recognized on a conscious level.

This is why surveys and interviews based on individual's opinion are not enough to find out users' satisfaction. The impacts that happen in a subconscious level may not be revealed in a survey answer. But with brain scans like PET (positron emission tomography) and fMRI (functional magnetic resonance imaging), it is possible to see more clearly the brain's reaction to each stimulus received by the environment and to understand in details how behavior is affected.

Every space - natural or built - will be interpreted by the brain differently. Some features of the environment might be interpreted as a symbol of power and show a hierarchical position, some will cause surprise and awe, others will bring back memories, or stimulate learning and alertness. Both Systems-I and II-can process those messages. However, the impacts on System I, which controls most of the mental processes, are much larger than System II, which only has a conscious perception of the environment. All this will reflect on the behavior 
and well-being of people.

The recognition of known and unknown spaces, for instance, is a behavior that was developed during evolution. Animals that did not have alertness levels raised when in an unfamiliar territory had a higher chance of being attacked by a predator. That is the reason why the territoriality is an instinctive behavior that the brain is hardwired to present. When an individual recognizes a space as his own territory, the attention levels tend to drop. As a result, it will be easier to relax and this might impact behavior.

Patients with Alzheimer who have privative rooms with their own personal objects for decoration have a much calmer behavior, less aggressive and anxious. When patients are allowed to have their own personal decoration, a link is made with their histories, and things become familiar. As a consequence of feeling "at home", stress levels get lower [7].

Contrastingly, spaces that are interpreted as threatening by System I, for instance, will activate automatic responses of fight or flight behavior, raising stress levels. Environments that show hierarchy and power tend to inhibit spontaneous behavior. On the other hand, spaces that stimulate spontaneity will bring out more creativity and collaboration among their users.

Spaces also generate expectations, which are a powerful tool to change brain and behavior. Placebo effect is an example of how expectations can unconsciously alter the brain. Depression, pain and sleep disorders can often have conditions changed by the placebo effect. Patients suffering from such problems can feel improvement in their symptoms even when taking pills that, unbeknownst to them, have no active substance. How can architecture generate expectations that improve well-being?

On the other hand, it must be considered that spaces will not necessarily affect everyone the same way. Neither can it be considered that with neuroarchitecture architects will be able to create "the perfect room" where brains will all work at their best.
The brain may be hardwired to present some innate behaviors, but it is also shaped by the culture and experiences individuals have during their lives. This means that architects must always consider their target public when designing a building. If in a culture marble is a symbol of power, a building made of marble will impact people differently than in a culture where marble is a symbol of poverty.

In addition, architects must always consider the function their building will have. A great architecture for a hospital will not be great for a school. Neither will a classroom be a good surgery room. Each space has a specific purpose and, consequently, must have a different design.

Therefore, neuroarchitecture application goes way beyond understanding the brain to create more efficient spaces. It is fundamental that architects understand the users of the building as well as the purpose of such building and each space inside of it. Only when considering these three factors together (brain knowledge, building purpose and user) architects will be able to design buildings for Systems I and II.

\section{Priming the Brain through Architecture}

"The design of a physical place influences the mental state of the people in that space. That shapes their attitudes and behavior" [8].

The retina captures information from the environment and sends them to the brain even when individuals are not directly looking anywhere. It works like this for all senses. Even when people are not consciously paying attention to sounds, textures or smells, the senses are capturing information and sending them to the brain. In its turn, the brain will process this information, but there will not necessarily be any conscious perception of it, unless it is something relevant, like the smell of food that activates appetite or the sound of a bug flying that provokes the instinctive reaction of head deviating, for example.

When a stimulus impacts System I and not System II, 
it is possible to influence people without them even realizing it. The technique, known as "priming", involves introducing a stimulus - a word, an image, a sound or a design - that has an effect on a person's later behavior, even if they cannot remember the stimulus in the first place.

For instance, a study in a British university tested how images can prime people for certain behaviors [9]. The experiment consisted in having an "honesty box" in an office kitchen where staff could pay for the tea and coffee they consumed. There was a suggested price list on the wall. Above the price list, the researchers posted a new image each week for 10 weeks, alternating between images of flowers and images of human eyes. Over the ten-week period, the researchers found that when there were the eyes' images above the price list, staff were primed and paid almost three times as much as when there were only flowers images.

This is an example of how by simply switching an image on the wall changed individuals' behavior. But how can that be applied to architecture?
Recent studies at universities in Canada and USA [10] suggest that the height of the ceiling affects the ability of problem solving. A room with high ceiling gives the brain a sensation of freedom, stimulating creativity and spontaneous behavior. On the other hand, a room with low ceiling makes the brain more focused and concentrated. For example, in retail spaces, low ceiling helps consumers to be more attentive to product defects.

In 2009, The Fun Theory (Rolighetsteorin), a campaign initiative from Volkswagen Sweden-created by DDB Stockholm showed an interesting way of influencing behavior: it presented a series of clever "design interventions" aimed at influencing behavior through making things "fun to do". One of the challenges was to prime people to use the stairs instead of the escalators. Simply telling people that it is healthier to use the stairs is not very efficient. So, the stairs were turned into a giant piano keyboard, with audio accompaniment. There was a $66 \%$ raise on the number of people using the stairs.

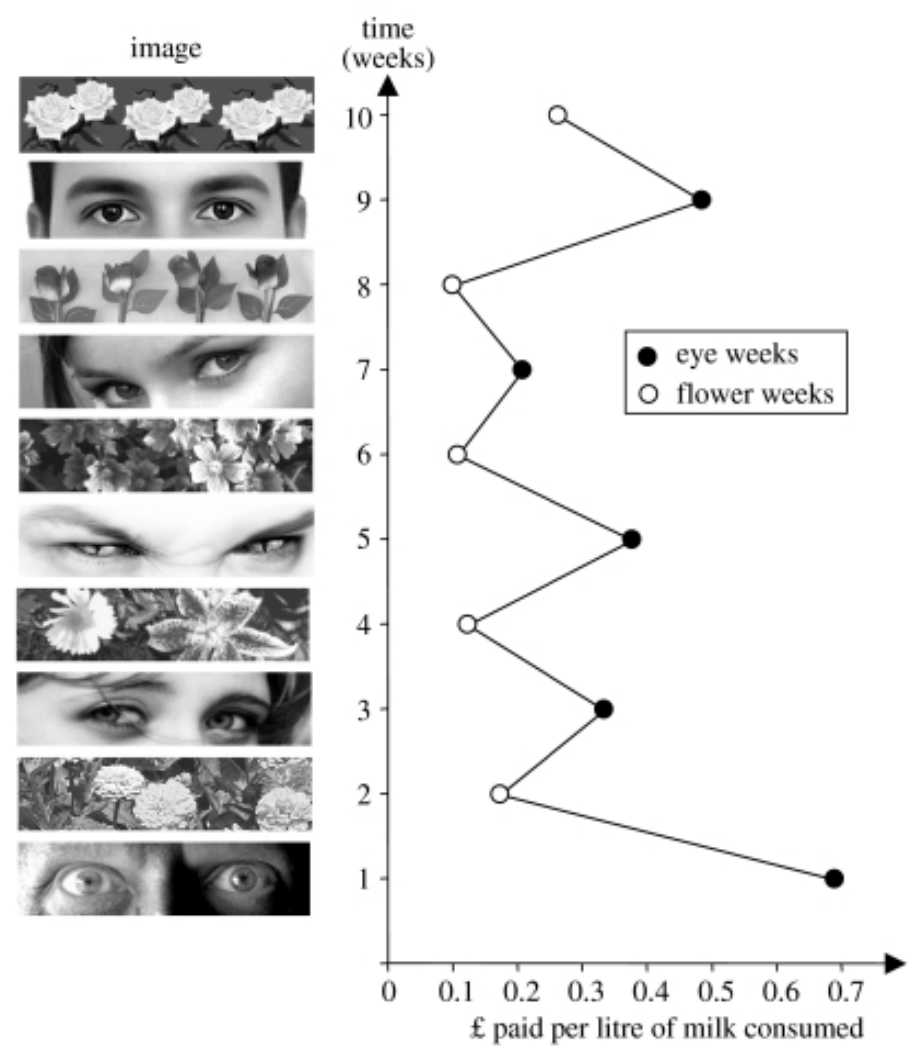

Fig. 1 Pounds paid per litre of tea/coffee consumed as a function of week and image type [9]. 


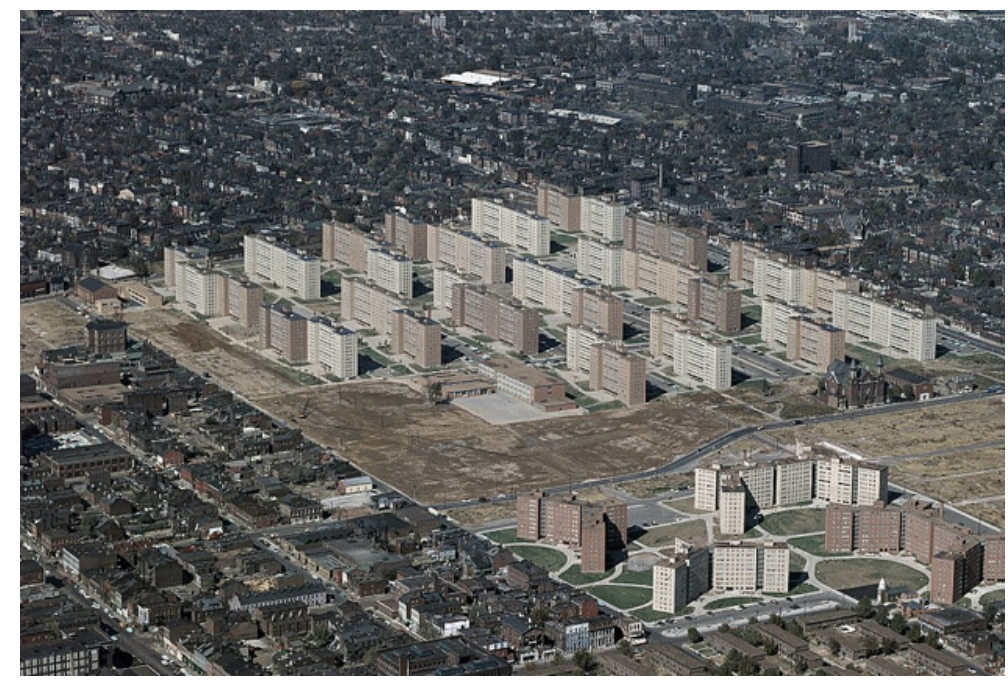

Fig. 2 Pruitt-Igoe Housing Project, St. Louis-Missouri [public domain image].

There are failure cases of priming through architecture as well. Pruitt-Igoe Housing Project of St. Louis, Missouri was built during the height of Modernism in the 1950s. This public housing complex was meant to have a rational architectural design that would house around 2,870 families. However, less than two decades after its construction, it was entirely demolished.

Pruitt-Igoe was initially considered by its residents as a very safe place and "an oasis in the desert" compared to the extremely poor quality of housing they had occupied previously. However, by the end of the 1960s, Pruitt-Igoe was nearly abandoned and had deteriorated into a decaying, dangerous, crime-infested neighborhood. How could that happen in such a short period of time? Have architectural aspects of the Pruitt-Igoe Housing Project contributed to that?

Priming is an important technique that can be very helpful to architects in both ways: firstly, to make sure buildings will not prime people to have negative behaviors; and secondly, to consider how buildings can prime people to present positive, healthier behaviors.

\section{Conclusion}

People nowadays usually spend $90 \%$ of their time inside buildings. The experience inside such spaces will have a direct impact on the brain. Consequently, behavior, performance and well-being might change according to the spaces individuals attend.

However, most of those behavior changes happen under the conscious level. System II-slow thinking - is responsible for conscious thoughts and for the analysis of new situations. But, to save energy, the brain was hardwired to work based mainly on automatic behaviors. System I-fast thinking-is responsible for instinctive, impulsive innate behaviors. Although humans are rational beings, the great majority of brain processes are unconscious and do not follow reason.

This is why people can be primed to change specific behaviors. Individuals can be primed when some environment information - image, sound, design - is noticed unconsciously and impacts the brain in a way to alter behavior without people noticing it.

In applying neuroarchitecture and designing to impact System I, architects can change the way buildings impact their users. Architecture can be used to reinforce cognition, memory, diminish stress and negative emotions. Furthermore, buildings can become even more efficient, resulting in schools that improve learning and memory, hospitals that improve recovery and so on. Neuroarchitecture is not meant to eliminate the architect's creativity and intuition, but to be one more tool to be used when designing a new project.

As great as neuroarchitecture findings can be, there is a crucial detail that must not be forgotten: there is not 
set of rules that lead to right or wrong answers. Each project must be done considering the specificity of each case. Individuals are unique according to their genetics, culture and their life experiences. Neuroarchitecture points to some reactions brains have to specific stimuli in neutral situations. To apply that, architects must be able to interpret them and analyze each case as unique.

\section{References}

[1] Ruskin, J. 1849. The Seven Lamps of Architecture. New York: John Wiley.

[2] Gonçalves, R., and Paiva, A. 2014. Triuno: As muitas Faces do seu Cérebro. São Paulo: Clube de Autores.

[3] Rock, D. 2009. Your Brain at Work. New York: Harper Business.

[4] Jung, C. G. 1970. "Collected Works." In Civilization in
Transition. Vol. 10. Princeton University Press.

[5] Kahneman, D. 2011. Thinking: Fast and Slow. New York: Farrar, Straus and Giroux.

[6] Churchil, W. 1943. "House of Commons Rebuilding." Accessed January 20, 2018. http://hansard.millbank systems.com/commons/1943/oct/28/house-of-commons-r ebuilding.

[7] Zeisel, J. 2006. Inquiry by Design. New York: W. W. Norton \& Company.

[8] Augustin, S. 2009. Place Advantage: Applied Psychology for Interior Architecture. New York: Wiley.

[9] Bateson, M., Nettle, D., and Roberts, G. 2006. "Cues of Being Watched Enhance Cooperation in a Real-World Setting." Accessed January 20, 2018. https://www.ncbi. nlm.nih.gov/pmc/articles/PMC1686213.

[10] Meyers-Levy, J., and Zhu, R. 2007. "The Influence of Ceiling Height: The Effect of Priming on the Type of Processing That People Use." Accessed November 24, 2017. https://assets.csom.umn.edu/ assets/71190.pdf. 